\title{
Development of a New Active Material Based on SiC-Fiber/Aluminum Composite
}

\author{
Hiroshi Asanuma ${ }^{1}$, Genji Hakoda ${ }^{1, *}$, Haruki Kurihara ${ }^{1, *}$ and Yun Lu $^{2}$ \\ ${ }^{1}$ Department of Electronics and Mechanical Engineering, Faculty of Engineering, Chiba University, Chiba 263-8522, Japan \\ ${ }^{2}$ Department of Urban Environment Systems, Faculty of Engineering, Chiba University, Chiba 263-8522, Japan
}

\begin{abstract}
The present paper proposes an active $\mathrm{SiC}$-fiber/aluminum composite utilizing its thermal deformation caused by non-uniform distribution of reinforcement fibers. A laminate of a continuous and unidirectional SiC fiber reinforced aluminum plate and an unreinforced aluminum plate was fabricated by the interphase forming/bonding method using a copper insert foil and its thermal deformation characteristics were investigated. The fabricated composite curved unidirectionally in the fiber direction by cooling from the hot pressing temperature, which is different from the behavior of a bimetal. Curvature of the composite at room temperature was maximized by investigating the effect of thickness of the aluminum plate, distance between the fibers and length of the composite in the experimented range. Under the optimum condition, its dependence on fiber length was clarified. It was also revealed that copper diffused but concentrated around the fibers, and that the interphase forming/bonding method with a copper insert increased the curvature of the composite. In a thermal cycling test, the curvature of the composite at room temperature reduced by heating from that to zero at the temperature of about $580 \mathrm{~K}$. The zero-curvature temperature and the curvature at room temperature were reproducible for ten thermal cycles. These results suggest an availability of this composite as an active material.
\end{abstract}

(Received September 3, 2004; Accepted December 20, 2004)

Keywords: aluminum, meal matrix composite, active material, actuator, smart material

\section{Introduction}

The new field "Smart Materials and Structural Systems")" has recently been receiving much attention. In this field, shape changes of structural materials have been attempted by embedding actuator materials such as shape memory alloys and piezoelectric ceramics. ${ }^{2,3)}$ These actuating materials are called "Active Materials."

Asanuma has been proposing thermal deformation type active composites as simple and effective active materials without such functional materials as shape memory alloys and piezoelectric ceramics. ${ }^{4)}$ As shown in Fig. 1, the proposed concept mainly consists of thermal deformation caused by non-uniform distribution of reinforcing fibers, and generation of heat for actuation and sensing of deformation

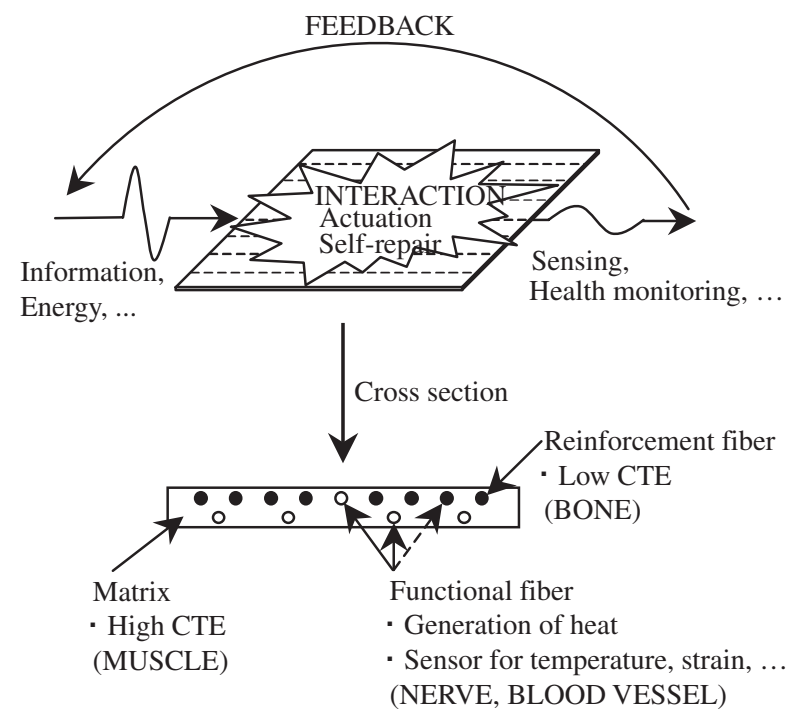

Fig. 1 Concept of the active composite. for shape control by embedded reinforcing and/or functional fibers. If the material system was regarded as a living body, the matrix would play the role of muscle, the reinforcement fiber the role of bone, and the functional fiber and sometimes the reinforcing fiber would also play the role of blood vessel and/or nerve.

As a fundamental work to realize the material system, Asanuma developed active CFRP (carbon fiber reinforced plastics)/aluminum laminates first, ${ }^{5)}$ where the aluminum having a high CTE (coefficient of thermal expansion) works as muscles, and the CFRP having a nearly zero CTE, especially the carbon fiber itself having a negative CTE, works as bone. In addition, the carbon fiber works as blood vessel to supply energy to the materials system for actuation because it efficiently generates electric resistant heat. Then, the concept was applied to $\mathrm{SiC}$-fiber/aluminum composites to realize higher temperature active composites, that is, thermal deformation of SiC-fiber reinforced aluminum composites caused by non-uniform distribution of the SiCfiber was advantageously examined, and their new application was found out. ${ }^{6)}$ After this development, Lu et al. ${ }^{7)}$ showed a possibility of using the carbon core of the embedded SiC-fiber as a heating element, which is mostly the same as the carbon fiber in the active CFRR/aluminum laminates introduced above.

A major difference of the active SiC-fiber/aluminum composites from bimetals is anisotropy of thermal deformation due to the directionality of the continuous SiC-fibers. By taking advantage of the deformation, design and control of it become available. This positive usage of thermal deformation in the field of structural materials will be able to develop a light weight and high strength planar actuator. A simple and useful actuation is unidirectional bending of a light-weight structural material panel. Thermal deformation of structural materials is regarded as a negative phenomenon in general, but it is positively used in this study. There are no previous researches from this standpoint except the authors' researches. This active material system will be able to replace a part 
of conventional mechanical systems because of simplification and weight reduction by removal of complicated and heavy actuation systems, and making them free from lubrication and wear problems by removal of joints, so it will be used for aerospace and many other mechanical applications. In addition, if the other functions of aluminum such as high electrical and thermal conductivities are taken into consideration, more applications will be found.

In this study, the proposed active material based on $\mathrm{SiC}$ fiber/aluminum composites was fabricated and its actuation was examined by experiments to investigate the effect of fiber distribution on maximization of deformation, reproducibility of deformation during heating cycles, the effect of fiber length on thermal deformation, and other aspects. In order to add smart functions to this material system, the prior researches such as embedment of fiber optic sensor ${ }^{8)}$ are needed to be combined as a next step.

\section{Experimental}

\subsection{Materials}

0.2 to $0.8 \mathrm{~mm}$ thick, $30 \mathrm{~mm}$ wide and $60 \mathrm{~mm}$ long pure aluminum plate (A1050P-O), SiC-fiber $(0.14 \mathrm{~mm}$ diameter, produced by Textron Systems, type SCS-2) of which CTE (coefficient of thermal expansion) is much smaller than that of aluminum, and $0.01 \mathrm{~mm}$ thick copper foil of $99.9 \%$ purity were used as matrix, reinforcement and insert, respectively. $0.2 \mathrm{~mm}$ thick titanium plate of $99.5 \%$ purity of which CTE is much smaller than that of aluminum was used to make a titanium-aluminum bimetal as a reference.

\subsection{Fabrication of composites}

The materials mentioned above were arranged as shown in Fig. 2 to obtain SiC-fiber/aluminum composites with nonuniform distribution of the SiC-fibers by the "Interphase Forming/Bonding Method (IF/B Method). ${ }^{9)}$ " The applied hot-pressing conditions were the temperature of $873 \mathrm{~K}$, the pressure of $2.7 \mathrm{MPa}$ and the period of $1.2 \mathrm{ks}$ in a vacuum of $1 \times 10^{2} \mathrm{~Pa}$. The characteristic of the IF/B Method can be briefly explained using Fig. 2 as follows: The matrix plates and the insert material used for bonding of them react with each other and produce a liquid alloy, a part of which remains in the clearances between the reinforcement fibers and the U-
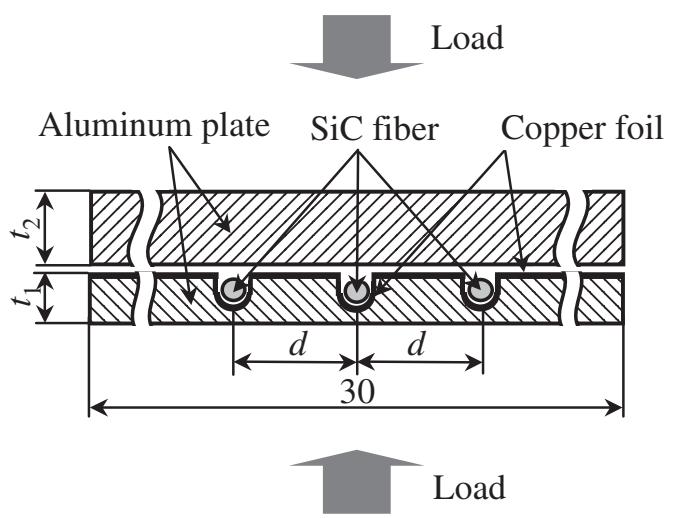

Fig. 2 Cross sections of the materials arranged to fabricate the SiC-fiber/ aluminum active composite by hot-pressing. grooves previously made for the alignment of the fibers, and the rest is squeezed out of the composite material. Using this technique, matrix bonding and coating of alloy on fiber can be realized at the same time. It enables embedding brittle fibers such as optical fibers in the composites to fabricate smart material systems as a next step of this research.

\subsection{Fabrication of reference materials}

To investigate the effect of the copper introduced around the $\mathrm{SiC}$-fiber in the aluminum matrix by the IF/B Method, $\mathrm{SiC}$-fiber/aluminum composites were made by conventional hot-pressing without using copper insert. The hot-pressing temperature was selected at $873 \mathrm{~K}$ which is high enough for diffusion bonding of aluminum plates, and the pressure was selected at 16.2 MPa which is high enough for plastic flow of aluminum at $673 \mathrm{~K}$. The hot-pressing period was widely changed from 1.2 to $19.2 \mathrm{ks}$ to investigate its effect.

In addition, the $0.2 \mathrm{~mm}$ thick titanium plate was laminated on the $0.2 \mathrm{~mm}$ thick aluminum to make a bimetal for comparison.

\subsection{Evaluation of the samples}

The hot-pressed samples were cooled in the furnace from hot-pressing temperature and their room temperature curvatures were measured. The cooling was undertaken after removal of hot-pressing pressure. The cooling rate at $873 \mathrm{~K}$ was about $0.03 \mathrm{~K} / \mathrm{s}$. The pressure applied on the sample by the punch of the die-set during cooling was as low as $2.7 \mathrm{kPa}$. The curvature of the sample $r^{-1}$ was evaluated as the average curvature shown in Fig. 3. Shapes of some samples were measured using a laser displacement sensor attached on a XY stage.

As maximization of the curvature $r^{-1}$ of the SiC-fiber/ aluminum active composite at room temperature leads to maximization of its performance, the effects of the aluminum plate thickness $t_{2}$, the SiC-fiber spacing $d$ (shown in Fig. 2), the specimen length $L$ and fiber length $l$ on the curvature $r^{-1}$ of the $\mathrm{SiC}$-fiber/aluminum active composite at room temperature were investigated under constant $t_{1}(=0.2 \mathrm{~mm})$.

Using the best sample obtained by the investigation above, temperature dependence of the curvature of the SiC-fiber/ aluminum active composite was examined by thermal cycles of heating in an electric furnace and cooling in air, where the temperature at which the sample becomes flat $\left(r^{-1}=0\right)$ and its curvature at room temperature at each cycle were monitored. The heating rate was kept constant at $0.2 \mathrm{~K} / \mathrm{s}$ and the cooling rate at the beginning of air-cooling $(580 \mathrm{~K})$ was about $1 \mathrm{~K} / \mathrm{s}$.

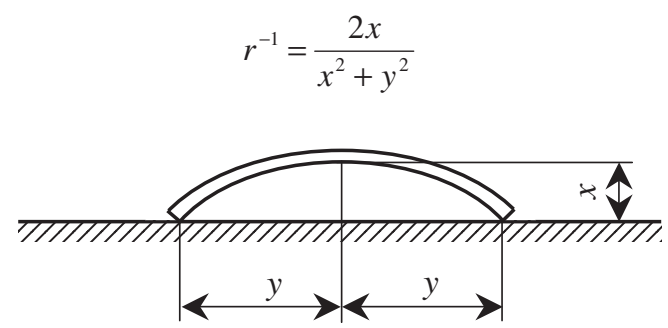

Fig. 3 Evaluation of average curvature of the SiC-fiber/aluminum active composites. 


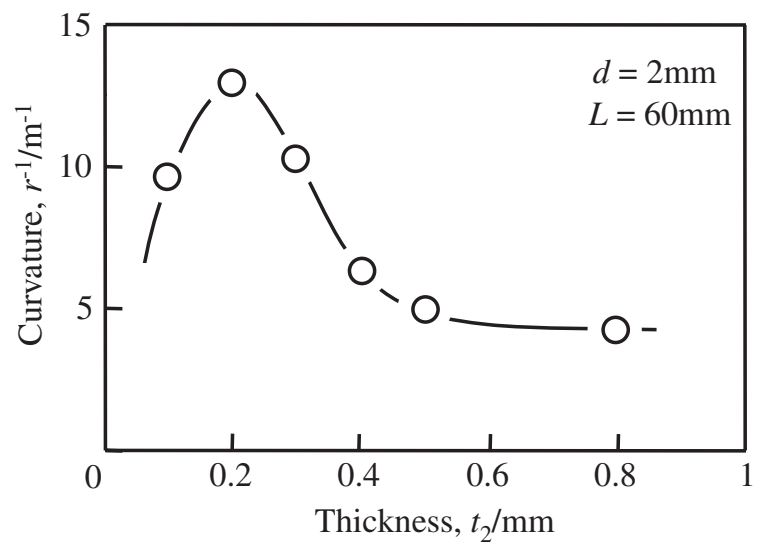

Fig. 4 Effect of thickness of aluminum plate $t_{2}$ on room temperature curvature of the SiC-fiber/aluminum active composite $r^{-1}$ under a constant distance between fibers $(d=2 \mathrm{~mm})$ and length of composite $(L=60 \mathrm{~mm})$.

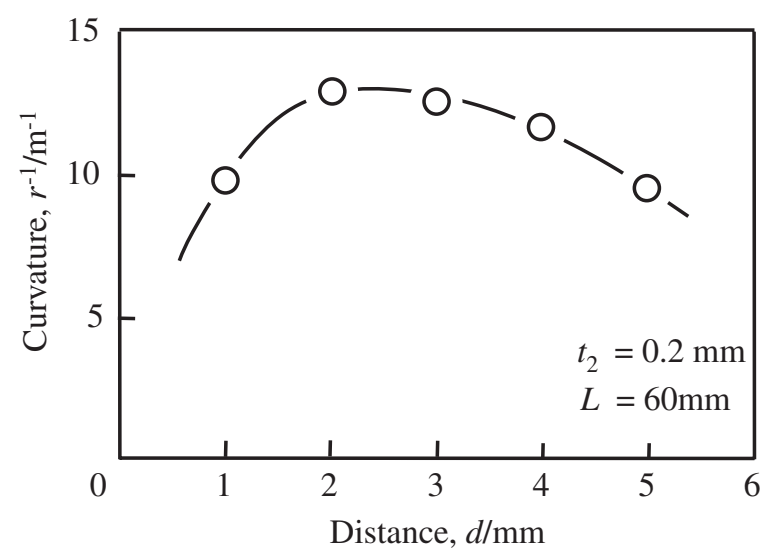

Fig. 5 Effect of distance between fibers $d$ on room temperature curvature $r^{-1}$ of the SiC-fiber/aluminum active composite under a constant thickness of aluminum plate $\left(t_{2}=0.2 \mathrm{~mm}\right)$ and length of composite $(L=60 \mathrm{~mm})$.

\section{Results and Discussion}

\subsection{Effect of aluminum thickness and SiC-fiber spacing on curvature of the composite}

In Fig. 4, the effect of the thickness of the unreinforced aluminum plate $t_{2}$ which was changed from 0.1 to $0.8 \mathrm{~mm}$ in this experiment on the curvature $r^{-1}$ of the SiC-fiber/ aluminum active composite at room temperature was shown when $d$ and $L$ were kept constant at $2 \mathrm{~mm}$ and $60 \mathrm{~mm}$, respectively. According to this figure, $r^{-1}$ is maximized at $t_{2}=0.2 \mathrm{~mm}$. The reason for the maximization is superimpose of the curvature increase caused by increase of bending moment and the curvature decrease caused by increase of bending rigidity with increasing $t_{2}$.

In Fig. 5, the effect of the SiC-fiber spacing $d$ on the curvature $r^{-1}$ of the SiC-fiber/aluminum active composite at room temperature was shown when $t_{2}$ and $L$ were kept constant at $0.2 \mathrm{~mm}$ and $60 \mathrm{~mm}$, respectively. According to this figure, $r^{-1}$ is maximized at $d=2 \mathrm{~mm}$. The reason for the maximization is superimposing of the curvature increase caused by decrease of bending rigidity and the curvature

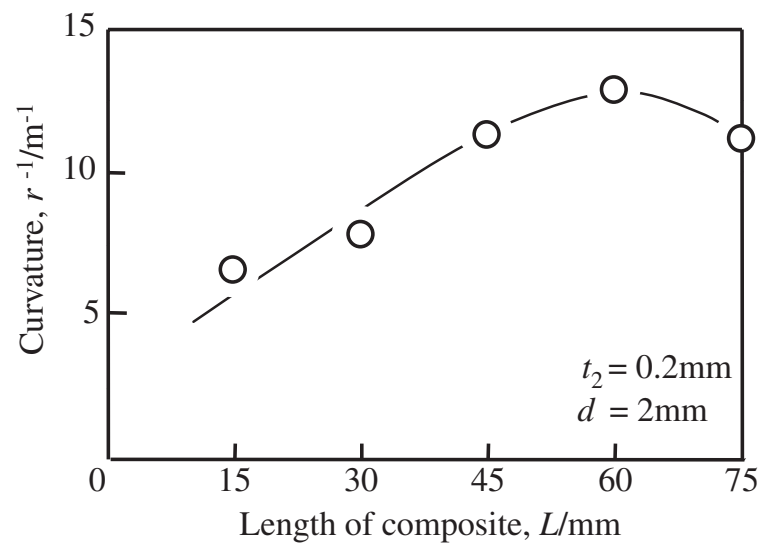

Fig. 6 Effect of composite length $L$ on room temperature curvature of the SiC-fiber/aluminum active composite $r^{-1}$ under constant thickness of aluminum plate $\left(t_{2}=0.2 \mathrm{~mm}\right)$ and distance between fibers $(d=2 \mathrm{~mm})$.

decrease caused by decrease of bending force due to decrease of fiber volume fraction with increasing $d$.

\subsection{Effect of specimen length and fiber length on curvature of the composite}

The curvature of the SiC-fiber/aluminum active composite specimen is considered to become smaller near the end of the specimen because the stress transfer between the fiber and the matrix becomes smaller. So the effect of specimen end on its average curvature should become larger with decreasing of $L$, and it was experimentally examined.

In Fig. 6, the result, that is, the effect of $L$ on $r^{-1}$ of the specimen at room temperature under constant $t_{2}(0.2 \mathrm{~mm})$ and $d(2 \mathrm{~mm})$ are shown. The figure shows that $r^{-1}$ increases with increasing $L$ up to around $60 \mathrm{~mm}$, but it rather decreases at around $75 \mathrm{~mm}$. The reason for this decrease is that, up to $60 \mathrm{~mm}$, the copper insert can be smoothly squeezed out of the specimen as $\mathrm{Al}-\mathrm{Cu}$ liquid alloy through the clearances between fiber and matrix by hot pressing, but beyond this length, the brittle $\mathrm{Al}-\mathrm{Cu}$ alloy comes to remain in the specimen and voids are also formed in it. For longer specimens, further investigations such as on hot-pressing condition are necessary.

By making positive use of the problem of the curvature reduction near the fiber ends, the average curvature of the active composite can be changed by changing the fiber length. So the effect of the fiber length $l$ on the average curvature $r^{-1}$ of the specimen under constant specimen length of $60 \mathrm{~mm}$ was investigated, and the effect was clarified as shown in Fig. 7. According to this figure, $r^{-1}$ decreases with decreasing $l$. So, the average curvature or its local curvature is proved to be changeable to some extent by changing the fiber length.

\subsection{Shape measurements}

Shapes of the SiC-fiber/aluminum active composite and the titanium/aluminum bimetal made as a reference are compared in Fig. 8, where their thicknesses are $0.350 \mathrm{~mm}$ and $0.375 \mathrm{~mm}$, respectively. According to this figure, the active composite is found to be bent only in the fiber direction whereas the bimetal has double curvatures. This result 


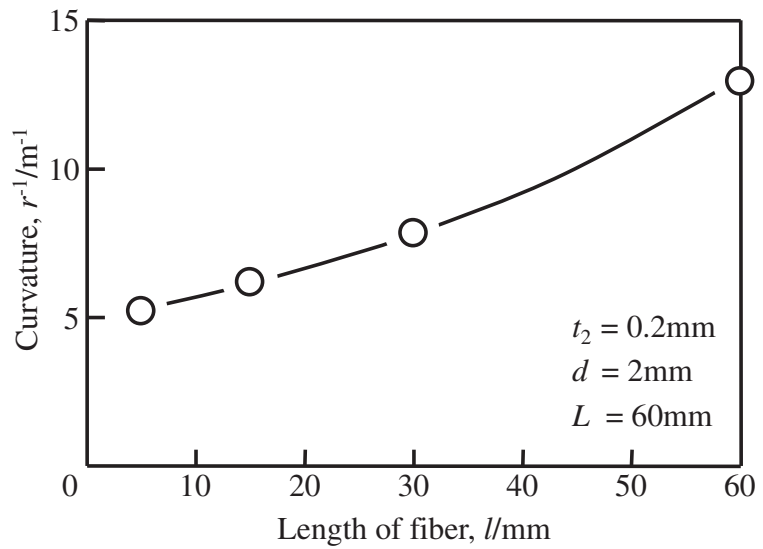

Fig. 7 Effect of fiber length $l$ on room temperature curvature of the SiCfiber/aluminum active composite $r^{-1}$ under constant thickness of aluminum plate $\left(t_{2}=0.2 \mathrm{~mm}\right)$, distance between fibers $(d=2 \mathrm{~mm})$ and length of composite $(L=60 \mathrm{~mm})$.
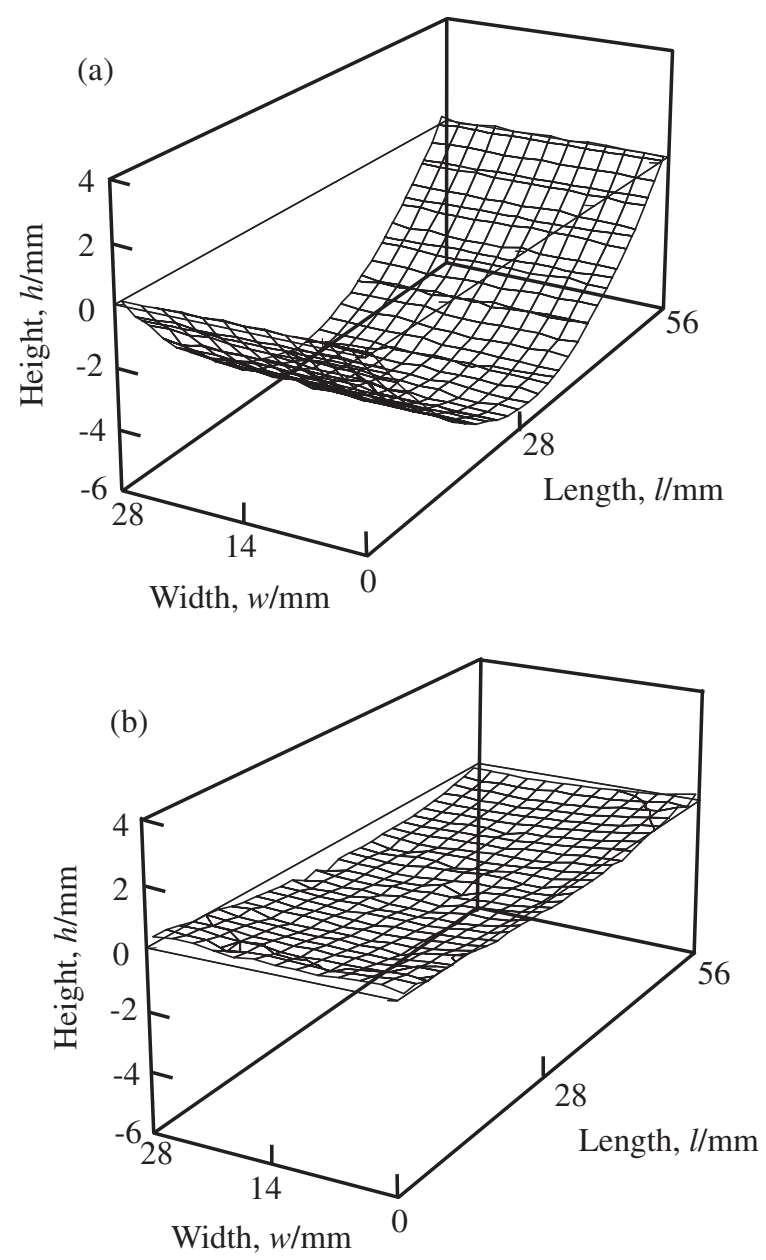

Fig. 8 Shapes of (a) the SiC-fiber/aluminum active composite and (b) the titanium/aluminum bimetal.

indicates the active composite is a new and useful engineering material different from conventional bimetals.

\subsection{Reproducibility of curvature of the composite after thermal cycles}

The active composite was heated in an electric furnace. In
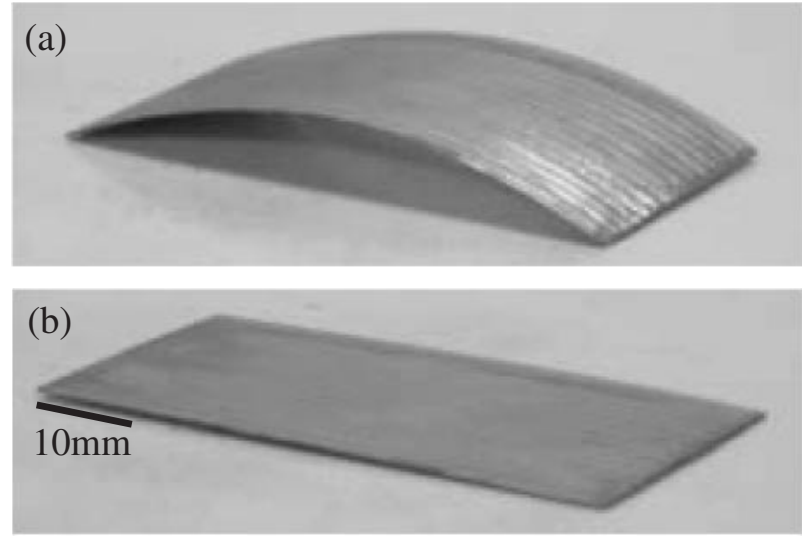

Fig. 9 Shapes of the SiC-fiber/aluminum active composite at (a) room temperature and (b) $580 \mathrm{~K}$.

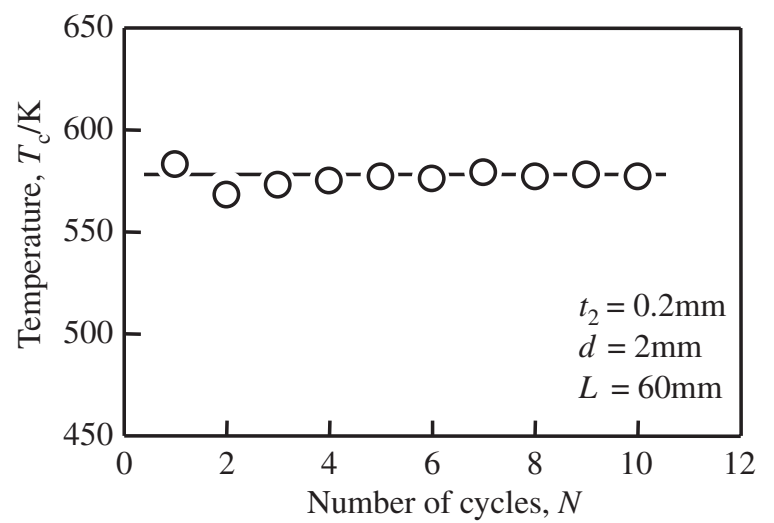

Fig. 10 Effect of number of heating cycles $N$ on temperature $T_{\mathrm{c}}$ where curvature of the $\mathrm{SiC}$ fiber/aluminum active composite becomes zero under constant thickness of aluminum plate $\left(t_{2}=0.2 \mathrm{~mm}\right)$, distance between fibers $(d=2 \mathrm{~mm})$ and length of composite $(L=60 \mathrm{~mm})$.

Fig. 9, the appearances of the specimen at R.T. and $580 \mathrm{~K}$ are shown. According to this figure, the curvature decreases by heating and becomes around zero at $580 \mathrm{~K}$. It was cooled down to R.T. and the curvature coincided well with its original value before this heating cycle.

As this critical temperature $T_{\mathrm{c}}$ where the curvature becomes zero was kept constant around $580 \mathrm{~K}$ even after 2 to 10 cycles as shown in Fig. 10, and the room temperature curvature was also kept constant after those cycles as shown in Fig. 11, repeatability of actuation of the composite was clarified. The little variations of the values during the first few cycles are caused by thermal residual stress induced during fabrication.

According to these results, thermal deformation of the composite is reproducible and it can be used as an active material.

\subsection{Effect of using copper insert on curvature of the composite}

In Fig. 12, a cross section of the active composite is shown with the results of EPMA line analyses for copper and aluminum along the line A-B. By the IF/B Method, the copper insert reacts with the aluminum matrix in several 


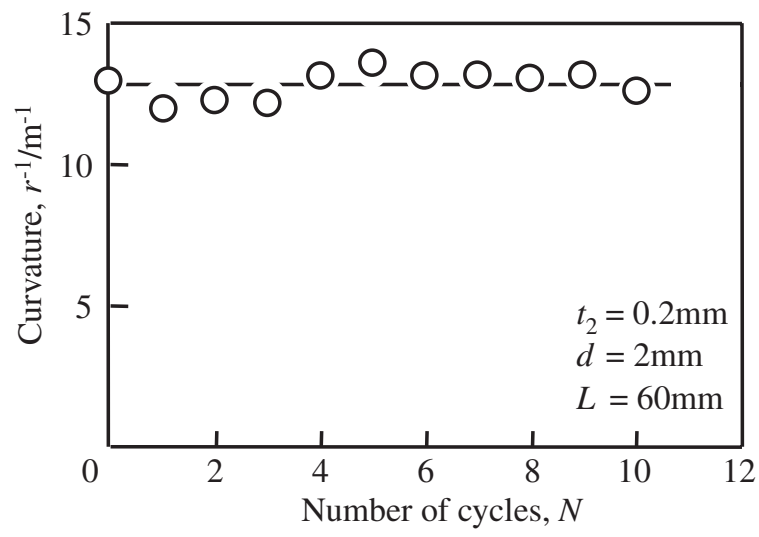

Fig. 11 Effect of number of heating cycles $N$ on room temperature curvature of the $\mathrm{SiC}$-fiber/aluminum active composite $r^{-1}$ under constant thickness of aluminum plate $\left(t_{2}=0.2 \mathrm{~mm}\right)$, distance between fibers $(d=2 \mathrm{~mm})$ and length of composite $(L=60 \mathrm{~mm})$.

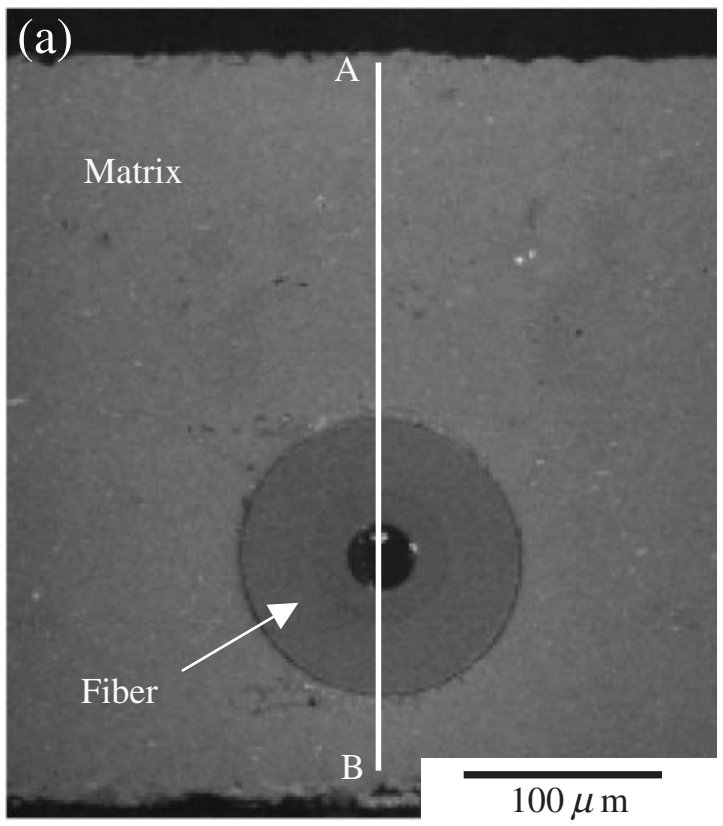

(b)

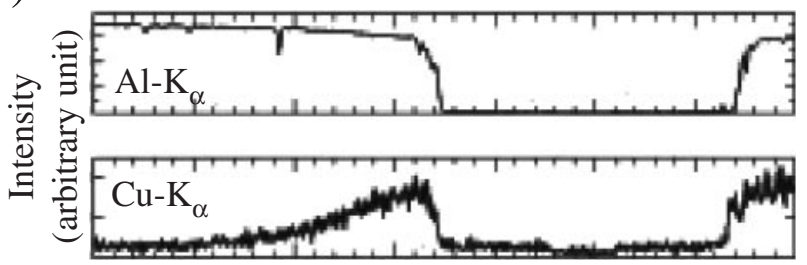

A

Fig. 12 SEM image of a cross section of the SiC fiber/aluminum active composite (a) and the result of EPMA line analysis of aluminum and copper along the line A-B shown in the SEM image (b).

seconds and comes into liquid, most of which is squeezed out of the specimen along the U-grooves made on the aluminum plate. According to this figure, it is clear that bonding line of the aluminum plates almost disappeared, and copper from the copper insert diffused but concentrated around the fiber.

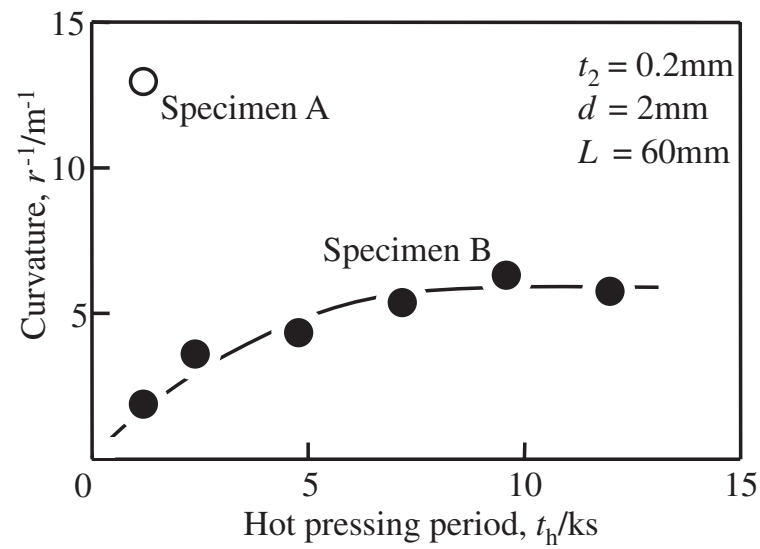

Fig. 13 Comparison of room temperature curvatures $r^{-1}$ of the SiC fiber/ aluminum active composites fabricated by the interphase forming/ bonding method using copper insert (Specimen A) and by normal hot pressing method (Specimen B) as a function of hot-pressing period $t_{\mathrm{h}}$.

In Fig. 13, the room temperature curvature of the active composite (Specimen A) is shown in comparison with those of Specimen B made without the copper insert as a function of hot-pressing time $t_{\mathrm{h}}$. According to this figure, the curvature of the Specimen B remains at lower values compared with that of the Specimen A even after being investigated under the wide range of hot-pressing period, which clarifies the advantage of the Specimen A even the difference of thickness, that is, those of the former and the latter are $0.35 \mathrm{~mm}$ and $0.40 \mathrm{~mm}$, respectively, is taken into consideration. The reasons for the advantage are thought that the copper introduced around the fibers by the IF/B Method increased the yield strength of the matrix around the fibers and the liquid phase produced by the reaction between the copper insert and the matrix enhanced bonding between the fibers and the matrix.

\section{Conclusions}

In this study, an active material was developed by making use of thermal deformation of SiC-fiber reinforced aluminum composites and the following results were obtained.

(1) As a laminate of a SiC-fiber reinforced aluminum plate and an unreinforced aluminum plate bent in the fiber direction during cooling from the hot-pressing temperature to R.T., it has the possibility of working as a new active material.

(2) Room temperature curvature of the active laminate, which reflects the activeness, strongly depends on thicknesses of the reinforced and the unreinforced parts, inter-fiber spacing, specimen length, fiber length and the state of fibermatrix interface. In the case of a mono-layer composite having a thickness of $0.2 \mathrm{~mm}$ for the reinforced part and a length of $60 \mathrm{~mm}$, its room temperature curvature can be maximized when the thickness of the unreinforced part and the fiber spacing are selected as $0.2 \mathrm{~mm}$ and $2 \mathrm{~mm}$, respectively, in the experimented range of this study.

(3) Near fiber ends, stress transfer between fiber and matrix is lowered, which reduces curvature of the composite. By making use of this mechanism, the curvature can be locally 
changed to some extent by changing the fiber length.

(4) When the active composite was heated, its curvature decreased with increasing temperature and became zero at around $580 \mathrm{~K}$. After cooling from this critical temperature, a cyclic heating from R.T. up to the critical temperature of $580 \mathrm{~K}$ was repeated, and it was found that the critical temperature and the room temperature curvature are reproducible up to 10 thermal cycles.

(5) The copper concentrated around the SiC-fibers of the active composites, which was introduced by the IF/B Method, increased the curvature of the active laminate.

\section{Acknowledgments}

A part of this research was supported by the Grant-in-Aid for Scientific Research on Priority Areas (B) by The Ministry of Education, Science, Sports and Culture under the area and contacting numbers of 725 and 11221204 , respectively.

\section{REFERENCES}

1) For example, T. Kishi: Proc. 1st Smart Materials Sympo., ed. by RIMCOF, (1999) pp. 1-3.

2) N. Hagood: Brochure for the MIT Active Materials \& Structures Laboratory, (1996)

3) F. Mezzanotti and M. Salvia: Proc. 4th ESSM and 2nd MIMR Conf., (Institute of Physics Publishing, Bristol, 1998) pp. 349-356.

4) H. Asanuma: JOM 52, 10 (2000) 21-25.

5) H. Asanuma, O. Haga, N. Naito and T. Tsuchiya: Proc. Annual Meeting of the Japan Society for Composite Materials, (1996) pp. 19-20.

6) H. Asanuma: Proc. JSME Annual Meeting, Vol. I, (1999) pp. 375-376.

7) Y. Lu, M. Hirohashi, H. Asanuma, R. Hayashi and J. Sakamoto: Proc. 29th FRP Sympo., ed. by The Society of Materials Science, (2000) pp. 299-302.

8) H. Asanuma, K. Ichikawa and T. Kishi: J. of Intelligent Material Systems and Structures 7 (1996) 307-311.

9) H. Asanuma, M. Hirohashi, M. Kase and T. Kikuchi: Proc. 1st Japan Intl. SAMPE Sympo., (Japan Chapter of SAMPE, 1989) pp. 979-984. 